\title{
The histone deacetylase inhibitor OBP-801 and eribulin synergistically inhibit the growth of triple-negative breast cancer cells with the suppression of survivin, Bcl-xL, and the MAPK pathway
}

\author{
Hisako Ono ${ }^{1,2} \cdot$ Yoshihiro Sowa $^{1}$ D $\cdot$ Mano Horinaka ${ }^{1} \cdot$ Yosuke lizumi $^{1} \cdot$ Motoki Watanabe $^{1} \cdot$ Mie Morita $^{1}$. \\ Emi Nishimoto $^{1} \cdot$ Tetsuya Taguchi $^{2} \cdot$ Toshiyuki Sakai $^{1}$
}

Received: 4 January 2018 / Accepted: 3 May 2018 / Published online: 11 May 2018

(c) The Author(s) 2018

\begin{abstract}
Purpose Triple-negative breast cancer (TNBC) is the most aggressive subtype of breast cancer. Eribulin was approved for the treatment of metastatic breast cancer through the EMBRACE trial, and a subgroup analysis in this clinical trial indicated the efficacy of eribulin in patients with TNBC. However, the prognosis of patients with TNBC is still poor due to various molecular characteristics. Therefore, there is an urgent need for a more effective treatment for the management of TNBC.

Methods We investigated the synergistic effect of a novel histone deacetylase (HDAC) inhibitor, OBP-801, and eribulin in TNBC cell lines because OBP-801 has been known to enhance the anti-tumor activities of other chemotherapeutic agents. The cell growth was analyzed, and the flow cytometry analysis was conducted to evaluate the effects on cell cycle and the induction of apoptosis. The mechanism underlying the enhancement of inhibition of TNBC cell growth was investigated through Western blot analyses.

Results The combination treatment of OBP-801 with eribulin showed the synergistic inhibition of the growth in TNBC cells, involved with the enhancement of apoptosis. We, for the first time, found that eribulin upregulated survivin and also that OBP-801 could remarkably suppress the upregulation of survivin by eribulin. Moreover, this combination potently suppressed Bcl-xL and the MAPK pathway compared with either agent alone.

Conclusion We found that the combination of OBP-801 and eribulin synergistically inhibited the growth with apoptosis in TNBC cells, suggesting that this combination might be a promising novel strategy for treating TNBC patients.
\end{abstract}

Keywords Triple-negative breast cancer $\cdot$ Eribulin $\cdot$ Histone deacetylase inhibitor $\cdot$ OBP-801 $\cdot$ Apoptosis

\begin{abstract}
Abbreviations
TNBC Triple-negative breast cancer

HDAC Histone deacetylase

ER Estrogen receptor
\end{abstract}

Electronic supplementary material The online version of this article (https://doi.org/10.1007/s10549-018-4815-x) contains supplementary material, which is available to authorized users.

Yoshihiro Sowa

ysowa@koto.kpu-m.ac.jp

1 Department of Molecular-Targeting Cancer Prevention, Graduate School of Medical Science, Kyoto Prefectural University of Medicine, Kawaramachi-Hirokoji,

Kamigyo-ku, Kyoto 602-8566, Japan

2 Department of Endocrine and Breast Surgery, Graduate School of Medical Science, Kyoto Prefectural University of Medicine, Kawaramachi-Hirokoji, Kamigyo-ku, Kyoto 602-8566, Japan

$\begin{array}{ll}\text { HER2 } & \text { Human epidermal growth factor receptor } 2 \\ \text { DMSO } & \text { Dimethyl sulfoxide } \\ \text { FBS } & \text { Fetal bovine serum } \\ \text { PgR } & \text { Progesterone receptor } \\ \text { CCK-8 } & \text { Cell Counting Kit-8 } \\ \text { CI } & \text { Combination index } \\ \text { AIF } & \text { Apoptosis-inducing factor } \\ \text { cDNA } & \text { Complementary DNA } \\ \text { SD } & \text { Standard deviation } \\ \text { ZEB1 } & \text { Zinc finger E-box binding homeobox 1 }\end{array}$

\section{Introduction}

Systemic therapy has important roles in breast cancer management. Therapy decisions are based on the subtypes of invasive ductal carcinoma of the breast. Since triple-negative breast cancer (TNBC) has no major effective target 
molecules, most patients must receive cytotoxic chemotherapy. However, the prognosis of TNBC is poor, perhaps due to heterogeneity [1]. Therefore, a new effective therapy is desired for patients with TNBC.

Eribulin is a well-tolerated cytotoxic drug in the management of metastatic breast cancer. A phase 3 randomized clinical trial (EMBRACE trial) showed that eribulin significantly improved the overall survival, and it has been approved for the treatment of refractory and metastatic breast cancer in Japan, the US, and Europe [2]. A subgroup analysis of this clinical trial also indicated that eribulin was more effective in patients with TNBC than estrogen receptor (ER)-positive breast cancer [3]. We administer eribulin as a monotherapy or in combination with anti-human epidermal growth factor receptor 2 (HER2) antibodies [4], and a clinical trial of eribulin and the anti-PD-1 antibody pembrolizumab therapy for metastatic TNBC is now in progress (ClinicalTrial.gov Identifier: NCT02513472). The combination therapy using eribulin might have the potential to improve the prognosis of patients with TNBC.

Histone deacetylase (HDAC) inhibitors are epigenometargeting drugs that have been approved for the treatment of cutaneous and peripheral T-cell lymphoma and multiple myeloma. HDAC inhibitors are reported to enhance the anti-tumor activities of other chemotherapeutic agents $[5,6]$. Various clinical trials of combination therapies of HDAC inhibitors have been carried out. For breast cancer, the combination of the HDAC inhibitor entinostat with the non-steroidal aromatase inhibitor exemestane for ER-positive breast cancer significantly improved the overall survival [7], receiving a breakthrough therapy designation from the FDA. A clinical trial of entinostat and the anti-PD-L1 antibody atezolizumab for TNBC is also in progress (ClinicalTrial.gov Identifier: NCT02708680). OBP-801, originally known as spiruchostatin $\mathrm{A}$, as an enhancer of plasminogen activator inhibitor-1 gene expression [8], was identified as a novel HDAC inhibitor in our previous study using p21 promoter-reporter screening [9]. OBP-801 showed the most potent HDAC inhibitory activity in our study, being about 50 times more effective than the HDAC inhibitor vorinostat [9]. This novel HDAC inhibitor OBP-801 is now under clinical trial in the US (ClinicalTrial.gov Identifier: NCT02414516).

In order to find a new strategy for the treatment of TNBC with a poor prognosis, we investigated whether OBP-801 could enhance the inhibitory effect of eribulin on human TNBC cells. We expected that OBP-801 known to suppress survivin $[10,11]$ was an appropriate agent for the combination with eribulin when eribulin was proved to upregulate survivin in TNBC like other microtubule dynamics inhibitors [12]. Our findings showed that the combination treatment of OBP-801 and eribulin induced the synergistic growth inhibition of TNBC cells through apoptosis with the suppression of Bcl-xL and the MAPK pathway as well as survivin. The combination therapy of OBP-801 and eribulin might be a promising treatment for patients with TNBC.

\section{Materials and methods}

\section{Reagents}

Eribulin was purchased from Eisai Co., Ltd. (Tokyo, Japan), and OBP-801 was provided by Oncolys BioPharma Inc. (Tokyo, Japan). These agents were dissolved in dimethyl sulfoxide (DMSO). The pan-caspase inhibitor zVAD-fmk was purchased from R\&D Systems (Minneapolis, MN) and also dissolved in DMSO. DMSO and these agents were diluted $1: 1000$ in culture medium.

\section{Breast cancer cell lines and cell culture}

Human breast cancer SUM159PT cells were purchased from Asterand Bioscience (Detroit, MI); MDA-MB-231, MCF-7, and Hs-578-t cells were obtained as cell lines of NCI-60 from the NCI Developmental Therapeutics Program (Bethesda, MD); and MDA-MB-436 cells were obtained from the American Type Culture Collection (Manassas, VA). SUM159PT cells were maintained in Ham's F-12 medium supplemented with $5 \%$ fetal bovine serum (FBS), $1 \mu \mathrm{g} / \mathrm{ml}$ hydrocortisone, and $5 \mu \mathrm{g} / \mathrm{ml}$ insulin at $37{ }^{\circ} \mathrm{C}$ in $5 \% \mathrm{CO}_{2}$. MDA-MB-231 cells were cultured in RPMI 1640 medium with $10 \% \mathrm{FBS}, 2 \mathrm{mM}$ glutamine, 50 units/ml penicillin, and $100 \mu \mathrm{g} / \mathrm{ml}$ streptomycin at $37{ }^{\circ} \mathrm{C}$ in $5 \% \mathrm{CO}_{2}$. MCF-7, Hs578-t, and MDA-MB-436 cells were maintained in DMEM medium supplemented with 10\% FBS, $4 \mathrm{mM}$ glutamine, 50 units $/ \mathrm{ml}$ penicillin, and $100 \mu \mathrm{g} / \mathrm{ml}$ streptomycin at $37^{\circ} \mathrm{C}$ in 5\% $\mathrm{CO}_{2}$. SUM159PT, MDA-MB-231, Hs-578-t, and MDAMB-436 cells do not express ER, progesterone receptor (PgR), or HER2, while MCF-7 cells express a high level of ER, low level of PgR, and no detectable level of HER2.

\section{Cell growth assay}

Cell growth was evaluated using the Cell Counting Kit- 8 (CCK-8) (Dojindo, Kumamoto, Japan), as previously reported [13].

\section{Combination index}

The combination index (CI) values were calculated using the CalcuSyn software program (Biosoft, Great Shelford, UK). Synergism is defined as more than the expected additive effect with a $\mathrm{CI}<1.0$. 


\section{Detection of apoptosis and cell cycle analyses}

SUM159PT and MDA-MB-231 cells treated with agents were harvested by trypsinization, centrifuged, and suspended in PBS containing $0.1 \%$ Triton X-100 and $150 \mu \mathrm{g} /$ $\mathrm{ml}$ RNase A, and the nuclei were stained with $50 \mu \mathrm{g} / \mathrm{ml}$ propidium iodide. The DNA content in stained nuclei was analyzed by FACSCalibur (Becton Dickinson, Franklin Lakes, NJ). The hypodiploid population (sub-G1) was quantified using the CellQuest software program (Becton Dickinson) to detect apoptosis, while the ModFit LT Ver2.0 software package (Verity Software House, Topsham, ME) was used to analyze the cell cycle.

\section{Western blot analyses}

SUM159PT and MDA-MB-231 cells treated with agents were lysed with RIPA buffer (50 mM Tris- $\mathrm{HCl}$ [pH 8.0], $150 \mathrm{mM} \mathrm{NaCl}, 1 \% \mathrm{NP}-40,0.5 \%$ deoxycholic acid, $0.1 \%$ SDS, $1 \mathrm{mM}$ DTT, and $0.5 \mathrm{mM}$ PMSF) or lysis buffer (50 mM Tris- $\mathrm{HCl}[\mathrm{pH} 8.0], 1 \% \mathrm{SDS}, 2 \mu \mathrm{g} / \mathrm{mL}$ aprotinin, $1 \mathrm{mM}$ DTT, and $0.5 \mathrm{mM}$ PMSF) and sonicated. When nuclear extracts were prepared from the cells treated with agents, Nuclear Extract Kit was used (Active Motif, Carlsbad, CA). Western blotting was performed, as previously reported [13]. Primary antibodies were rabbit anti-acetyl histone H3 (9649; Cell Signaling Technology, Danvers, MA), anti-histone H3 (9715; Cell Signaling Technology), anti-survivin (AF886; R\&D Systems), anti-Bcl-xL (2762; Cell Signaling Technology), anti-Bcl-2 (ab32124; Abcam, Cambridge, UK), anti-Mcl-1 (4572; Cell Signaling Technology), anti-Bax (sc-493; Santa Cruz Biotechnology, Dallas, TX), anti-Bad (9268; Cell Signaling Technology), anti-Bim (2933; Cell Signaling Technology), anti-phosphorylated ERK (9101; Cell Signaling Technology), anti-ERK (9102; Cell Signaling Technology), and anti-apoptosis-inducing factor (AIF) (ab32516; Abcam) antibodies, as well as mouse anti-XIAP (MAB822; R\&D Systems) and anti- $\beta$-actin (A5441; Sigma-Aldrich, St. Louis, MO) antibodies.

\section{Quantitative real-time RT-PCR}

Total cellular RNA extraction from SUM159PT and MDAMB-231 cells and complementary DNA (cDNA) synthesis were performed as previously reported [13]. A quantitative real-time RT-PCR analysis was performed with the cDNA and TaqMan probes (Thermo Fisher Scientific, Waltham, MA) to survivin (Hs04194392_s1) and GAPDH (Hs02758991_g1).

\section{Colony formation assay}

Cells were seeded at a density of 200 cells per well in 6-well plates. After incubating for $24 \mathrm{~h}$, the cells were treated with each agent. The cells were fixed with $10 \%$ formalin and stained with $0.1 \%$ crystal violet after incubation for further 9 days. The number of colonies in each cell line was counted.

\section{Statistical analyses}

Data are represented as the mean \pm standard deviation (SD) in triplicate. Statistical analyses were performed using a oneway analysis of variance (ANOVA) followed by Bonferroni post hoc tests. Samples were considered significantly different at $P<0.05$.

\section{Results}

\section{Eribulin and the HDAC inhibitor OBP-801 synergistically inhibit the growth of TNBC cells with apoptosis}

Since clinical data have indicated that eribulin is more effective for treating patients with TNBC than ER-positive breast cancer [3], we initially evaluated the inhibition of cell growth by eribulin in several human TNBC cell lines compared with ER-positive breast cancer MCF-7 cells. Eribulin inhibited the growth of the TNBC cell lines in a dose-dependent manner, and the result was more effective than that for MCF-7 cells (Supplementary Fig. 1a). The $\mathrm{IC}_{50}$ value of eribulin on TNBC SUM159PT cells was $8.93 \mathrm{nM}$, and that on TNBC MDA-MB-231 cells was $0.90 \mathrm{nM}$ (Fig. 1a). The HDAC inhibitor OBP-801 also inhibited the growth of the TNBC cell lines in a dose-dependent manner, and the degree of inhibition was more effective than that for MCF-7 cells (Supplementary Fig. 1b). The $\mathrm{IC}_{50}$ value of OBP-801 on SUM159PT cells was $8.82 \mathrm{nM}$, and that on MDA-MB-231 cells was 5.49 nM (Fig. 1a).

Since HDAC inhibitors are known to potentiate other chemotherapeutic agents $[5,6]$, we investigated whether OBP-801 enhanced the effectiveness of eribulin against TNBC cells. As shown in Fig. 1b, the growth of TNBC cells was synergistically inhibited by the combination treatment in comparison to either agent alone in SUM159PT and MDA-MB-231 cells. The CI values of this combination treatment were much less than 1.0 in both TNBC cells, thus indicating a synergistic effect (Fig. 1b). We also evaluated the dose-dependent activity in each combination ratio. The combination treatment of eribulin and OBP-801 inhibited the growth of the both TNBC cells in a dose-dependent manner (Fig. 1c). 


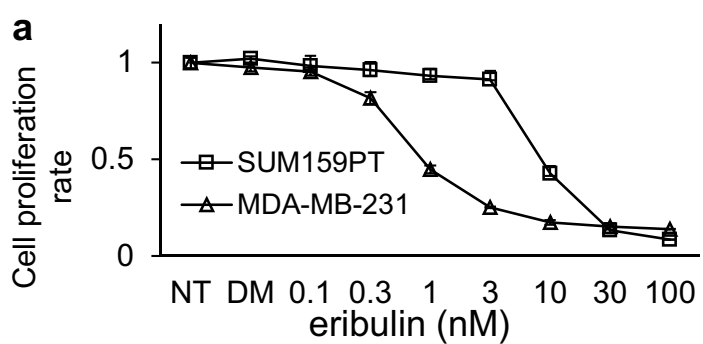

\begin{tabular}{|c|c|}
\hline cell lines & $\mathrm{IC}_{50}$ (eribulin) \\
\hline SUM159PT & $8.93 \mathrm{nM}$ \\
\hline MDA-MB-231 & $0.90 \mathrm{nM}$ \\
\hline
\end{tabular}

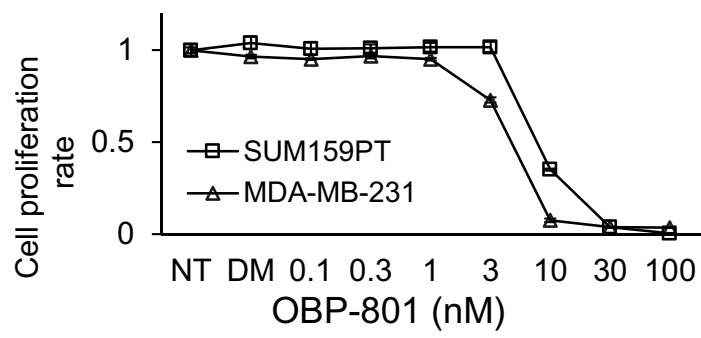

\begin{tabular}{|c|c|}
\hline cell lines & IC $_{50}$ (OBP-801) \\
\hline SUM159PT & $8.82 \mathrm{nM}$ \\
\hline MDA-MB-231 & $5.49 \mathrm{nM}$ \\
\hline
\end{tabular}
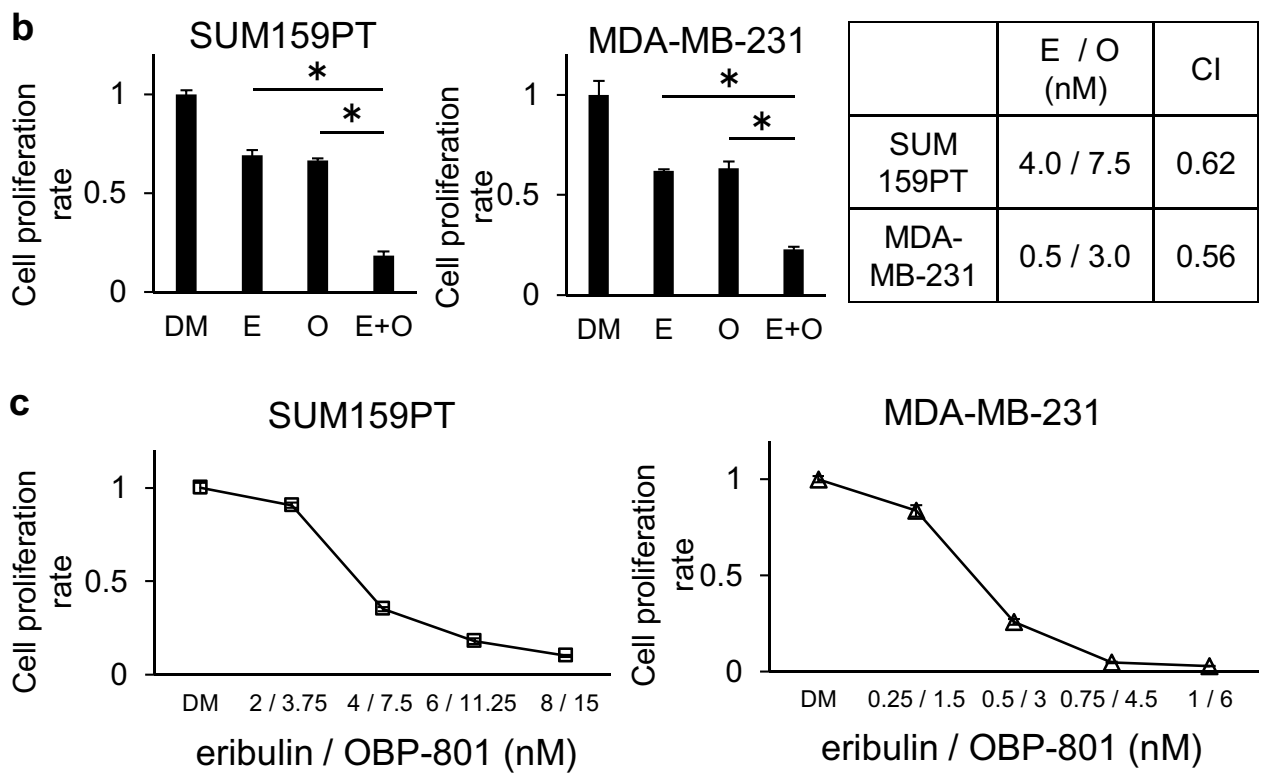

Fig. 1 Eribulin and OBP-801 synergistically inhibit the growth of TNBC cells. a SUM159PT and MDA-MB-231 cells were treated with the indicated concentrations of eribulin and OBP-801 for $72 \mathrm{~h}$. $N T$ non-treated, $D M$ treated with DMSO. The inhibition of cell growth was evaluated using CCK-8. The cell proliferation rate of each treatment was calculated by comparison with the NT samples. b SUM159PT cells were treated with $4.0 \mathrm{nM}$ eribulin and $7.5 \mathrm{nM}$ OBP-801, while MDA-MB-231 cells were treated with $0.5 \mathrm{nM}$ eribulin and $3.0 \mathrm{nM}$ OBP-801. $D M$ treated with DMSO, $E$ treated with eribulin, $O$ treated with OBP-801, $E+O$ treated with the combination of eribulin and OBP-801. SUM159PT cells were treated with DMSO or eribulin and/or OBP-801 for $72 \mathrm{~h}$, and MDA-MB-231 cells were

similarly treated for $96 \mathrm{~h}$. The inhibition of the cell growth was evaluated using CCK-8. The cell proliferation rate of each treatment was calculated by comparing the DMSO samples. The CI was calculated using CalcuSyn. Columns, mean values of triplicate data; bars, SD. $* P<0.05$. c Combination treatment of eribulin and OBP-801 inhibited the growth of TNBC cells in a dose-dependent manner. $D M$ treated with DMSO. SUM159PT or MDA-MB-231 cells were treated with the indicated concentrations of eribulin and OBP-801 for 72 or $96 \mathrm{~h}$, respectively. The inhibition of cell growth in the combination was evaluated using CCK-8. The cell proliferation rate of each treatment was calculated by comparison with the DM samples

We next examined the effect of this combination treatment on the cell cycle of TNBC cells. As a result, OBP-801 enhanced the G2/M arrest induced by eribulin in MDAMB-231 cells, but not in SUM159PT cells (Fig. 2a). The

common finding for this combination treatment on both TNBC cells was an enhancement of apoptosis (Fig. 2b), and the apoptosis was partially dependent on caspase pathway (Fig. 2c). 



Fig. 2 Combination treatment of eribulin and OBP-801 inhibits the growth with apoptosis in TNBC cells. SUM159PT cells were treated with $4.0 \mathrm{nM}$ eribulin and $7.5 \mathrm{nM}$ OBP-801, while MDA-MB-231 cells were treated with $0.5 \mathrm{nM}$ eribulin and $3.0 \mathrm{nM}$ OBP-801. $D M$ treated with DMSO, $E$ treated with eribulin, $O$ treated with OBP-801, $E+O$ treated with the combination of eribulin and OBP-801. a The TNBC cells were treated with DMSO or eribulin and/or OBP-801 for $72 \mathrm{~h}$, and the cell cycle populations were analyzed by flow cytometry. The cell cycle populations of combination treatment were compared

\section{Combination treatment of eribulin and OBP-801 enhances apoptosis with the suppression of survivin and $\mathrm{BCl}-\mathrm{xL}$}

In order to clarify the mechanisms underlying the enhancement of caspase-dependent apoptosis in the combination treatment, we investigated the effect of this treatment on apoptosis-related proteins. At first, OBP-801 increased the acetylation of histone $\mathrm{H} 3$, indicating that OBP-801 possessed an HDAC inhibitory activity under this treatment condition (Fig. 3a). The treatment of eribulin alone upregulated the expression of survivin, one of the anti-apoptotic molecules, at the protein level in both SUM159PT and MDA-MB-231 cells. On the other hand,

with those for each single treatment. b The TNBC cells were treated with DMSO or eribulin and/or OBP-801 for $72 \mathrm{~h}$, and the sub-G1 population was analyzed by flow cytometry. The sub-G1 population of combination treatment was compared with that of each single treatment. $\mathbf{c}$ The TNBC cells were treated with DMSO or the combination of eribulin and OBP-801 with or without $20 \mu \mathrm{M}$ zVAD-fmk for $72 \mathrm{~h}$. $Z$ zVAD-fmk. The sub-G1 population of combination treatment was compared by the presence of zVAD-fmk. Columns, mean values of triplicate data; bars, SD. $* P<0.05$

the treatment of OBP-801 alone clearly downregulated survivin in both TNBC cells. It was interesting to note that OBP-801 markedly suppressed the expression of survivin upregulated by eribulin at the protein and mRNA levels (Fig. 3a, b). Apart from that, Bcl-xL, one of the anti-apoptotic molecules, was more strongly suppressed by the combination treatment at the protein level than by OBP-801 alone in both cells, while Bcl-xL was not suppressed by eribulin alone (Fig. 3a). Taken together, these findings suggest that OBP-801 enhanced apoptosis by the suppression of survivin and $\mathrm{Bcl}-\mathrm{xL}$ in the combination treatment with eribulin against TNBC cells. In addition to the suppression of survivin and Bcl-xL, OBP-801 alone induced the expression of the apoptotic proteins, 
Fig. 3 Combination treatment of eribulin and OBP-801 downregulates survivin and $\mathrm{Bcl}-\mathrm{xL}$ in TNBC cells. SUM159PT cells were treated with $4.0 \mathrm{nM}$ eribulin and $7.5 \mathrm{nM}$ OBP-801, while MDA-MB-231 cells were treated with $0.5 \mathrm{nM}$ eribulin and $3.0 \mathrm{nM}$ OBP-801 in the same way as in Fig. 2. DM treated with DMSO, $E$ treated with eribulin, $O$ treated with OBP-801, $E+O$ treated with the combination of eribulin and OBP-801. a A Western blot analysis of acetylated histone H3 (Ace-His H3), histone H3, survivin, $\mathrm{Bcl}-\mathrm{xL}$, and other apoptosis-related proteins, including Bcl-2 family in TNBC cells treated with DMSO or eribulin and/or OBP-801 for $48 \mathrm{~h}$. b A real-time RT-PCR analysis of survivin in TNBC cells treated with DMSO or eribulin and/or OBP-801 for $48 \mathrm{~h}$. Values show the foldchange compared with DMSO treatment. Columns, mean values of triplicate data; bars, SD. $* P<0.05$ a

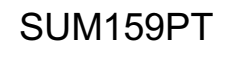

DM E O E+O

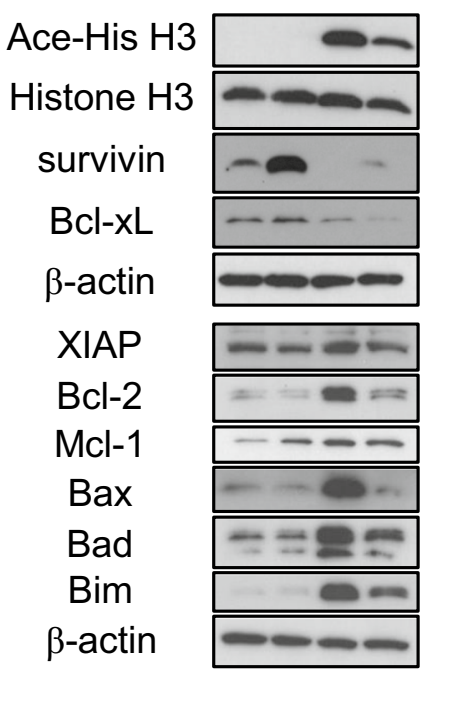

b $\quad \begin{gathered}\text { SUM159PT } \\ \text { survivin / GAPDH }\end{gathered}$

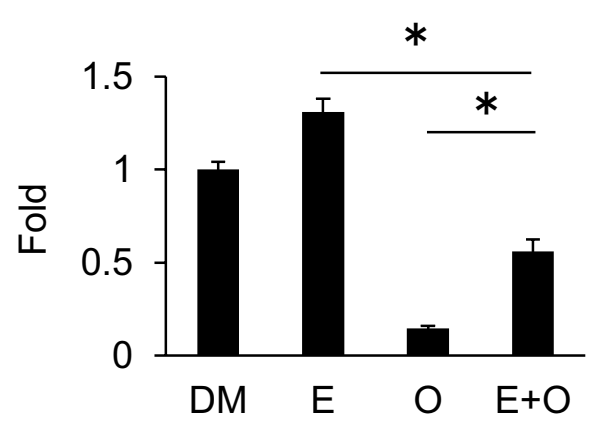
XIAP
MDA-MB-231

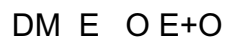

Ace-His H3

Histone $\mathrm{H} 3$ survivin

Bcl-XL $\beta$-actin

Bcl-2
Mcl-1
Bax
Bad
Bim
$\beta$-actin
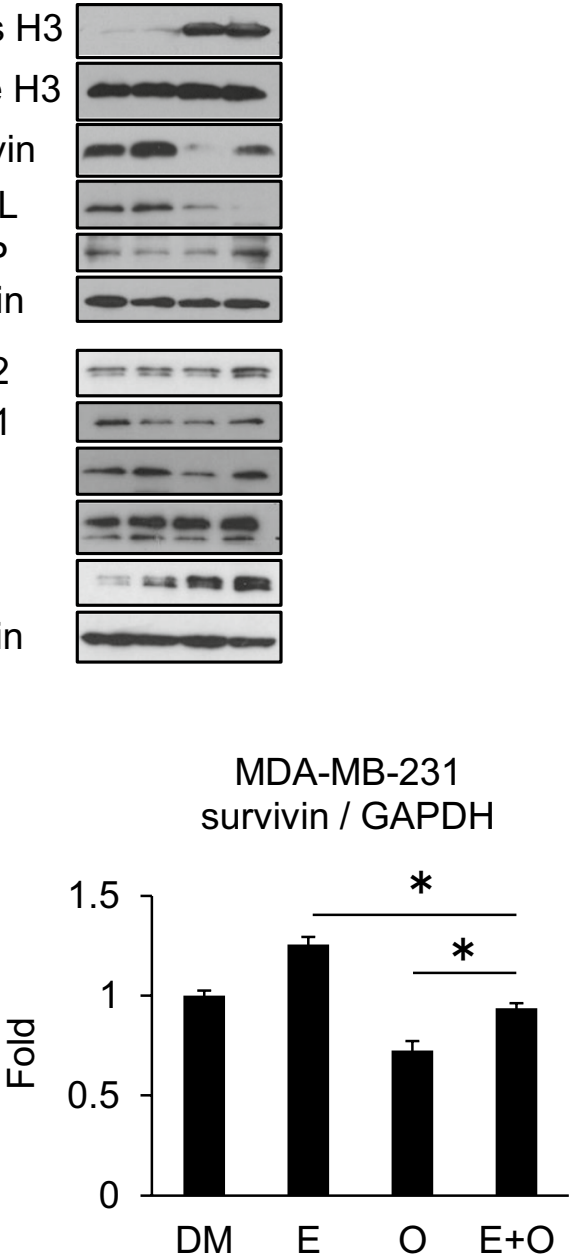

Bax, Bad, and Bim in SUM159PT and only Bim in MDAMB-231 cells (Fig. 3a). The induction of Bim by the combination treatment in both cells was not clearly stronger than OBP-801 alone, whereas that was obviously potent compared with that treated by eribulin alone (Fig. $3 \mathrm{a}$ ). It raises a possibility that the induction of Bim by OBP-801 partially contributes to the enhancement of apoptosis by eribulin in TNBC cells. We moreover investigated the mechanisms underlying the enhancement of caspase-independent apoptosis involved with AIF. AIF is one of the important proteins which an HDAC inhibitor leads caspase-independent apoptosis [14]. We evaluated that the translocation of AIF into the nucleus, but the expression of AIF in the nuclear extracts in the combination treatment was not higher than each agent alone in SUM159PT and MDA-MB-231 cells (Supplementary Fig. 2).

\section{Combination treatment of eribulin and OBP-801 also suppresses the MAPK pathway}

Next, we investigated the effects of the combination treatment on the MAPK pathway. Among microtubule dynamic inhibitors, vinca alkaloids are known to suppress the MAPK pathway [15], while paclitaxel enhances it in other carcinoma cells [16]. Therefore, we investigated whether eribulin enhanced or suppressed the MAPK pathway in TNBC cells. Eribulin was found to weakly decrease the phosphorylated ERK protein in SUM159PT and MDA-MB-231 cells (Fig. 4a). Given that HDAC inhibitors also reportedly suppress the phosphorylation of ERK in TNBC [17], we evaluated the effect of OBP-801 on the phosphorylation of ERK. A higher concentration of OBP-801 clearly decreased the phosphorylated ERK protein, but neither $7.5 \mathrm{nM}$ OBP-801 in SUM159PT cells nor $3.0 \mathrm{nM}$ 


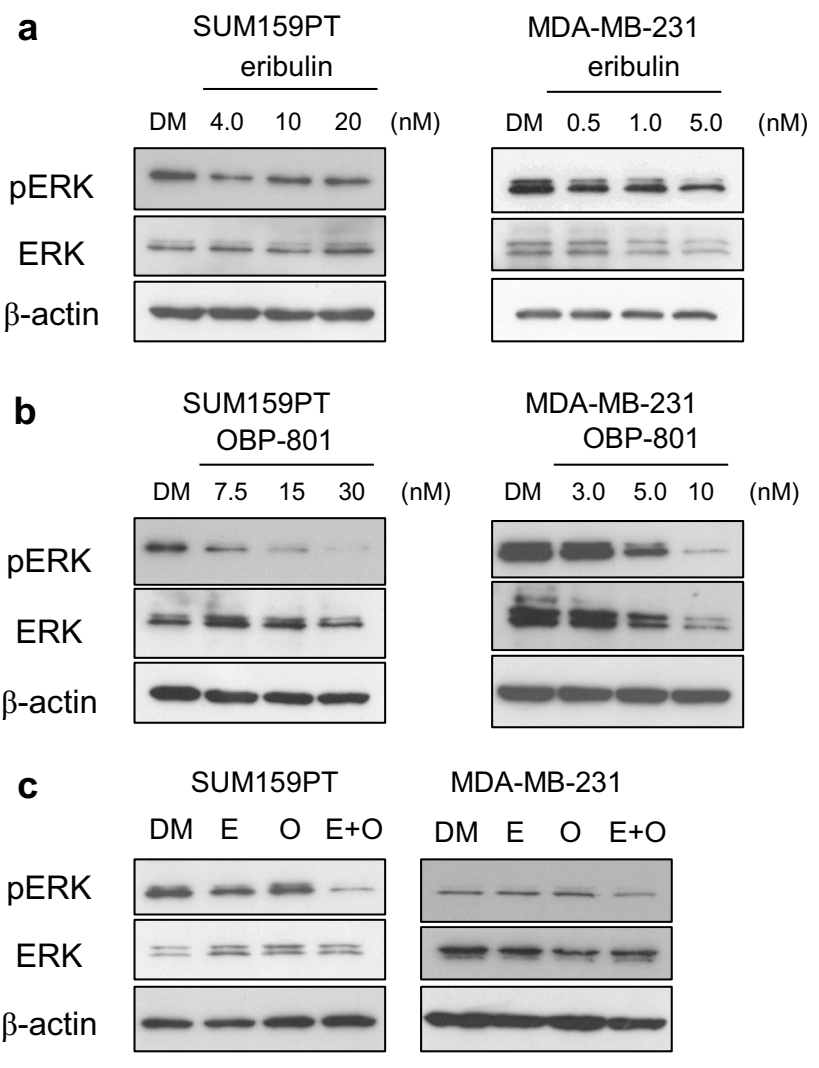

Fig. 4 Combination treatment of eribulin and OBP-801 suppresses the MAPK pathway in TNBC cells. a A Western blot analysis of phosphorylated ERK and total ERK in TNBC cells treated with the indicated concentrations of eribulin for $48 \mathrm{~h}$. b A Western blot analysis of phosphorylated ERK and total ERK in TNBC cells treated with the indicated concentrations of OBP-801 for $48 \mathrm{~h}$. c A Western blot analysis of phosphorylated ERK and total ERK in TNBC cells treated with DMSO or eribulin and/or OBP-801 for $48 \mathrm{~h}$. SUM159PT cells were treated with $4.0 \mathrm{nM}$ eribulin and $7.5 \mathrm{nM}$ OBP-801, while MDAMB-231 cells were treated with $0.5 \mathrm{nM}$ eribulin and $3.0 \mathrm{nM}$ OBP801 in the same way as in Figs. 2 and 3. DM treated with DMSO, $E$ treated with eribulin, $O$ treated with OBP-801, $E+O$ treated with the combination of eribulin and OBP-801. The image of $\beta$-actin in MDAMB-231 is the same as that in Fig. 3a due to the use of the same gel

OBP-801 in MDA-MB-231 cells sufficiently inhibited the MAPK pathway (Fig. 4b). Unexpectedly, the combination of eribulin with these low concentrations of OBP-801 in which we showed the synergistic effect (Fig. 1b), clearly decreased the phosphorylation of ERK (Fig. 4c). These results suggest that the inhibition of the MAPK pathway might be associated with the enhancement of apoptosis by the combination treatment against TNBC cells.

\section{Combination treatment of eribulin and OBP-801 also inhibits the growth of TNBC more potently than either agent alone in the long-term in vitro combination activity}

As an in vitro preclinical model, we also examined the combination activity in the long-term incubation. We performed colony formation assay of SUM159PT and MDA-MB-231 cells. As shown in Fig. 5a, b, the combination of eribulin and OBP-801 resulted in the significant inhibition of the colony formation more potently than either agent alone in the both TNBC cells. These data suggest that this combination treatment might be effective in vivo and clinical studies in TNBC models and TNBC patients.

\section{Discussion}

To identify a new strategy for the treatment of TNBC, we focused on eribulin, a well-tolerated chemotherapeutic agent against metastatic breast cancer that has promising efficacy, especially for TNBC [3]. Eribulin exerts its effects as a microtubule dynamic inhibitor via the different mechanism from other inhibitors such as taxanes and vinca alkaloids [18]. Eribulin is a unique microtubule dynamic inhibitor and has been recently shown to have several attractive effects on the microenvironment of breast cancer, such as reversing epithelial-mesenchymal transition $[19,20]$ and improving hypoxia in cancer [21,22].

Survivin has been implicated in not only the inhibition of apoptosis but also mitosis regulation [23, 24]. As shown in Fig. 3a, we found in our research that eribulin upregulated survivin in TNBC cells. To our knowledge, this is the first report to describe the upregulation of survivin by eribulin, while other microtubule inhibitors, paclitaxel and vincristine, were shown to enhance the expression of survivin [12]. Survivin is highly expressed in malignant tissues but it is not expressed in normal tissues [25], and the upregulation of survivin has been reported to have clinical importance as a poor prognostic marker in breast cancer [26-28]. We found a novel HDAC inhibitor OBP-801 to be a promising candidate for enhancing the effect of eribulin by suppressing survivin in TNBC (Fig. 3a). Since HDAC leads to the formation of transcriptionally silenced chromatin, HDAC inhibitors upregulate various silenced genes. However, they are also known to downregulate various genes. HDAC inhibitors were recently reported to suppress the promoter activity of survivin through AMPK and p38MAPK signaling, and Sp1 in colon cancer cells [29]. As shown in Fig. 3a, b, our data demonstrated that OBP-801 suppressed the expression of survivin at the protein and mRNA levels in TNBC cells, which is consistent with that. 
Fig. 5 Combination treatment of eribulin and OBP-801 inhibits TNBC cell growth more potently than either agent alone in colony formation assay. $D M$ treated with DMSO, $E$ treated with eribulin, $O$ treated with OBP-801, $E+O$ treated with the combination of eribulin and OBP-801. a SUM159PT cells were treated with DMSO or $4.0 \mathrm{nM}$ eribulin and/or $7.5 \mathrm{nM}$ OBP-801, and MDA-MB-231 cells were treated with DMSO or $0.5 \mathrm{nM}$ eribulin and/or $3.0 \mathrm{nM}$ OBP-801. After incubation for colony formation, the number of colonies was counted. The percent of colony numbers was calculated by comparing the DMSO samples. Columns, mean values of triplicate data; bars, SD. $* P<0.05$. b The representative images of stained colonies are also shown
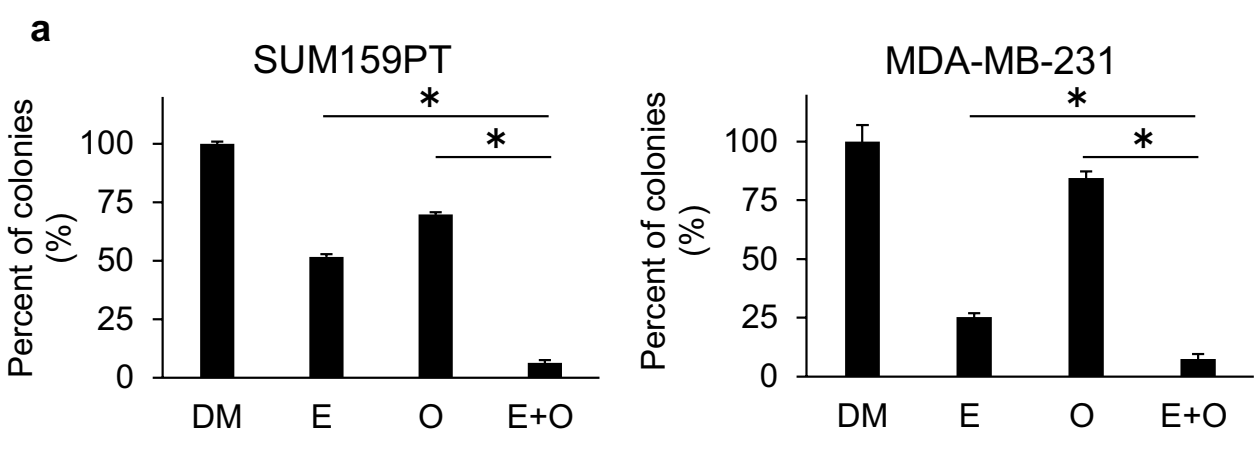

b
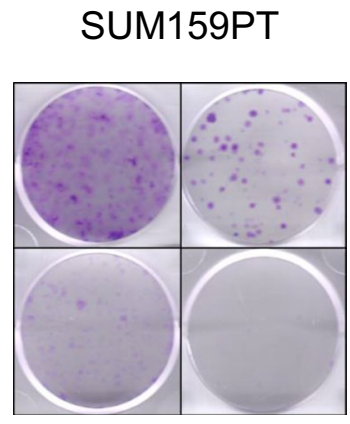

MDA-MB-231

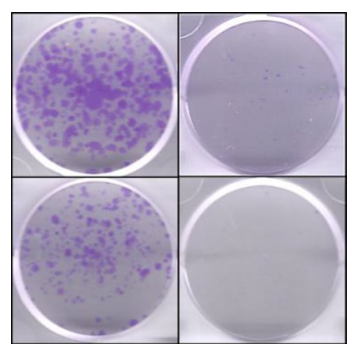

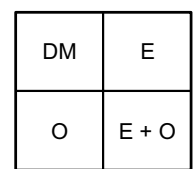

HDAC inhibitors have also been reported to induce apoptosis through the regulation of various anti- and/or pro-apoptotic molecules, such as Bcl-2 family molecules [30-33]. The mechanism underlying the suppression of $\mathrm{Bcl}-\mathrm{xL}$ protein by HDAC inhibitors has been reported to be regulated at the transcriptional or translational level in several carcinomas, including TNBC [32, 33]. In our research, the suppression of $\mathrm{Bcl}-\mathrm{xL}$ protein was more potently suppressed by the combination treatment than by OBP-801 alone in TNBC cells, while Bcl-xL was not suppressed by eribulin alone (Fig. 3a). Since other microtubule inhibitors were reported to induce the phosphorylation of Bcl-xL [34], the suppression of Bcl-xL in the combination treatment of OBP-801 and eribulin was thought to be resulted from various regulation at the transcriptional, translational, or posttranslational level. As shown in Supplementary Fig. 1b and other previous report [35], HDAC inhibitors have a potent anti-proliferative effect in TNBC cells. However, the administration of HDAC inhibitors in clinical as monotherapy is limited, and their therapeutic potential may lie in combination therapy with other chemotherapeutic agents [36]. In the present study, we showed that the combination treatment of OBP-801 and eribulin induced the synergistic growth inhibition of TNBC cells, partially through apoptosis. Eribulin proved to be a good partner for combination therapy with HDAC inhibitors.

The MAPK pathway is essential for cell proliferation, angiogenesis, and survival. The suppression of the MAPK pathway by the MEK inhibitor selumetinib was found to be effective in preventing lung metastasis of TNBC through the reversal of epithelial-mesenchymal transition, as reported in vitro and in vivo [37]. In patients with TNBC, the overexpression of ERK2 was found to indicate a high risk of death [38]. As shown in Fig. 4c, our results interestingly showed that the combination treatment of eribulin and OBP-801 suppressed the MAPK pathway more effectively than single agent alone in TNBC. Recently, vincristine has also been reported in lung cancer to downregulate the phosphorylated ERK protein, resulting in the suppression of the zinc finger E-box binding homeobox 1 (ZEB1), an activator of epithelial-mesenchymal transition [15]. Eribulin has been shown to reverse epithelial-mesenchymal transition both in vitro and in vivo $[19,20]$. Therefore, the suppression of the MAPK pathway by eribulin with OBP-801 in TNBC cells, as shown in our research, might efficiently suppress the ERK-ZEB1 pathway.

In conclusion, this is the first report to demonstrate that treatment with an HDAC inhibitor and eribulin inhibits growth with inducing apoptosis in TNBC cells. We believe that OBP-801 and eribulin are an effective combination therapy for treating TNBC.

Acknowledgements T. Sakai received the Grant/research funding (J152001034) from Oncolys BioPharma Inc. The English in this manuscript was reviewed by Brian Quinn (Japan Medical Communication Inc.). 


\section{Compliance with ethical standards}

Conflict of interest T. Sakai reports receiving commercial research grant from Oncolys BioPharma Inc. No potential conflicts of interest were disclosed by the other authors.

Open Access This article is distributed under the terms of the Creative Commons Attribution 4.0 International License (http://creativeco mmons.org/licenses/by/4.0/), which permits unrestricted use, distribution, and reproduction in any medium, provided you give appropriate credit to the original author(s) and the source, provide a link to the Creative Commons license, and indicate if changes were made.

\section{References}

1. Shah SP, Roth A, Goya R et al (2012) The clonal and mutational evolution spectrum of primary triple-negative breast cancers. Nature 486:395-399

2. Cortes J, O'Shaughnessy J, Loesch D et al (2011) Eribulin monotherapy versus treatment of physician's choice in patients with metastatic breast cancer (EMBRACE): a phase 3 open-label randomised study. Lancet 377:914-923

3. Twelves C, Cortes J, Vahdat L, Olivo M, He Y, Kaufeman PA, Awada A (2014) Efficacy of eribulin in women with metastatic breast cancer: a pooled analysis of two phase 3 studies. Breast Cancer Res Treat 148:553-561

4. Araki K, Fukada I, Yanagi H et al (2017) First report of eribulin in combination with pertuzumab and trastuzumab for advanced HER2-positive breast cancer. Breast 35:78-84

5. Wang L, Li H, Ren Y et al (2016) Targeting HDAC with a novel inhibitor effectively reverses paclitaxel resistance in non-small cell lung cancer via multiple mechanisms. Cell Death Dis 7:e2063

6. Chao MW, Lai MJ, Liou JP, Chang YL, Wang JC, Pan SL, Teng CM (2015) The synergic effect of vincristine and vorinostat in leukemia in vitro and in vivo. J Hematol Oncol 8:82

7. Yardley DA, Ismail-Khan RR, Melichar B et al (2013) Randomized phase II, double-blind, placebo-controlled study of exemestane with or without entinostat in postmenopausal women with locally recurrent or metastatic estrogen receptor-positive breast cancer progressing on treatment with a nonsteroidal aromatase inhibitor. J Clin Oncol 31:2128-2135

8. Masuoka Y, Nagai A, Shin-ya K et al (2001) Spiruchostatins A and $\mathrm{B}$, novel gene expression-enhancing substances produced by Pseudomonas sp. Tetrahedron Lett 42:41-44

9. Shindoh N, Mori M, Terada Y et al (2008) YM753, a novel histone deacetylase inhibitor, exhibits antitumor activity with selective, sustained accumulation of acetylated histones in tumors in the WiDr xenograft model. Int J Oncol 32:545-555

10. Toriyama S, Horinaka M, Yasuda S et al (2016) A histone deacetylase inhibitor, OBP-801, and celecoxib synergistically inhibit the cell growth with apoptosis via a DR5-dependet pathway in bladder cancer cells. Mol Cancer Ther 15:2066-2075

11. Yamada K, Horinaka M, Shinnoh M, Yoshioka T, Miki T, Sakai T (2013) A novel HDAC inhibitor OBP-801 and a PI3K inhibitor LY294002 synergistically induce apoptosis via the suppression of survivin and XIAP in renal cancer carcinoma. Int J Oncol 43:1080-1086

12. O'Connor DS, Wall NR, Porter AC, Altieri DC (2002) A p34 $4^{\text {cdc2 }}$ survival checkpoint in cancer. Cancer Cell 2:43-54
13. Ono H, Iizumi Y, Goi W, Sowa Y, Taguchi T, Sakai T (2017) Ribosomal protein S3 regulates XIAP expression independently of the NF- $\mathrm{\kappa B}$ pathway in breast cancer cells. Oncol Rep 38:3205-3210

14. Chen YJ, Wang WH, Wu WY et al (2017) Novel histone deacetylase inhibitor AR-42 exhibits antitumor activity in pancreatic cancer cells by affecting multiple biochemical pathways. PLoS ONE 22:e0183368

15. Chiu LY, Hsin IL, Yang TY et al (2017) The ERK-ZEB1 pathway mediates epithelial-mesenchymal transition in pemetrexed resistant lung cancer cells with suppression by vinca alkaloids. Oncogene 36:242-253

16. McDaid HM, Horwitz SB (2001) Selective potentiation of paclitaxel (taxol)-induced cell death by mitogen-activated protein kinase inhibition in human cancer cell lines. Mol Pharmacol 60:290-301

17. Plotkin A, Volmor CH, Wahlestedt C, Ayad N, El-Ashry D (2014) Transcriptional repression of ER through hMAPK dependent histone deacetylation by class I HDACs. Breast Cancer Res Treat 147:249-263

18. Jordan MA, Kamath K, Manna T, Okouneva T, Miller HP, Davis C, Littlefield BA, Wilson L (2005) The primary antimitotic mechanism of action of the synthetic halichondrin E7389 is suppression of microtubule growth. Mol Cancer Ther 4:1086-1095

19. Yoshida T, Ozawa Y, Kimura T et al (2014) Eribulin mesilate suppresses experimental metastasis of breast cancer cells by reversing phenotype from epithelial-mesenchymal transition (EMT) to mesenchymal-epithelial transition (MET) states. Br J Cancer 110:1497-1505

20. Terashima M, Sakai K, Togashi Y, Hayashi H, De Velasco MA, Tsurutani J, Nishio K (2014) Synergistic antitumor effects of $\mathrm{S}-1$ with eribulin in vitro and in vivo for triple-negative breast cancer cell lines. SpringerPlus 3:417

21. Funahashi Y, Okamoto K, Adachi Y et al (2014) Eribulin mesylate reduces tumor microenvironment abnormality by vascular remodeling in preclinical human breast cancer models. Cancer Sci 105:1334-1342

22. Ueda S, Saeki T, Takeuchi H, Shigekawa T, Yamane T, Kuji I, Osaki A (2016) In vivo imaging of eribulin-induced reoxygenation in advanced breast cancer patients: a comparison to bevacizumab. Br J Cancer 114:1212-1218

23. Li F, Ambrosini G, Chu EY, Plescia J, Tognin S, Marchisio PC, Altieri DC (1998) Control of apoptosis and mitotic spindle checkpoint by survivin. Nature 10:580-584

24. Giodini A, Kallio MJ, Wall NR et al (2002) Regulation of microtubule stability and mitotic progression by survivin. Cancer Res 62:2462-2467

25. Ambrosini G, Adida C, Altieri DC (1997) A novel anti-apoptosis gene, survivin, expressed in cancer and lymphoma. Nat Med 3:917-921

26. Hamy AS, Bieche I, Lehmann-Che J et al (2016) BIRC5 (survivin): a pejorative prognostic marker in stage II/III breast cancer with no response to neoadjuvant chemotherapy. Breast Cancer Res Treat 159:499-511

27. Ryan BM, Konecny GE, Kahlert S et al (2006) Survivin expression in breast cancer predicts clinical outcome and is associated with HER2, VEGF, urokinase plasminogen activator and PAI-1. Ann Oncol 17:597-604

28. Span PN, Sweep FC, Wiegerinck ET, Tjan-Heijnen VC, Manders P, Beex LV, de Kok JB (2004) Survivin is an independent prognostic marker for risk stratification of breast cancer patients. Clin Chem 50:1986-1993

29. Hsu YF, Sheu JR, Lin CH et al (2012) Trichostatin A and sirtinol suppressed survivin expression through AMPK and p38MAPK in HT29 colon cancer cells. Biochim Biophys Acta 1820:104-115 
30. Johnstone RW, Licht JD (2003) Histone deacetylase inhibitors in cancer therapy: Is transcription the primary target? Cancer Cell 4:13-18

31. Zhao Y, Tan J, Zhuang L, Jiang X, Liu ET, Yu Q (2005) Inhibitors of histone deacetylases target the Rb-E2F1 pathway for apoptosis induction through activation of proapoptotic protein Bim. Proc Natl Acad Sci 102:16090-16095

32. Chueh AC, Tse JWT, Dickinson M et al (2017) ATF3 repression of Bcl-xl determines apoptotic sensitivity to HDAC inhibitors across tumor types. Clin Cancer Res 23:5573-5584

33. Adams CM, Eischen CM (2016) Histone deacetylase inhibition reveals a tumor-suppressive function of MYC-regulated miRNA in breast and lung carcinoma. Cell Death Differ 23:1312-1321

34. Poruchynsky MS, Wang EE, Rudin CM, Blagosklonny MV, Fojo $\mathrm{T}$ (1998) Bcl-xL is phosphorylated in malignant cells following microtubule disruption. Cancer Res 58:3331-3338
35. Tate CR, Rhodes LV, Segar HC, Driver JL, Pounder FN, Burow ME, Collins-Burow BM (2012) Targeting triple-negative breast cancer cells with the histone deacetylase inhibitor panobinostat. Breast Cancer Res 14:R79

36. Bots M, Johnstone RW (2009) Rational combinations using HDAC inhibitors. Clin Cancer Res 15:3970-3977

37. Bartholomeusz C, Xie X, Pitner MK et al (2015) MEK inhibitor selumetinib (AZD6244; ARRY-142886) prevents lung metastasis in a triple-negative breast cancer xenograft model. Mol Cancer Ther 14:2773-2781

38. Bartholomeusz C, Gonzalez-Angulo AM, Liu P, Hayashi N, Lluch A, Ferrer-Lozano J, Hortobagyi GN (2012) High ERK protein expression levels correlate with shorter survival in triple-negative breast cancer patients. Oncologist 17:766-774 